\title{
ARTICLE
}

Cite this: DOI: $10.1039 /$ xoxxooooox

\section{Bridging Silicon Nanoparticles and Thermoelectrics: Phenylacetylene Functionalization}

Received ooth January 2012, Accepted ooth January 2012

DOI: $10.1039 / \times 0 x x 00000 x$

www.rsc.org/

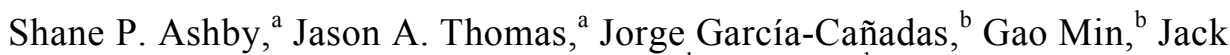 \\ Corps, ${ }^{\mathrm{c}}$ Anthony V. Powell, ${ }^{\mathrm{c}}$ Hualong Xu, ${ }^{\mathrm{d}}$ Wei Shen ${ }^{\mathrm{d}}$ and Yimin Chao*,
}

Silicon is a promising alternative to current thermoelectric materials $\left(\mathrm{Bi}_{2} \mathrm{Te}_{3}\right)$. Silicon nanoparticle based materials show especially low thermal conductivities due to the high number of interfaces, which increases the observed phonon scattering. The major obstacle with these materials is maintaining a high electrical conductivity. Surface functionalization with phenylacetylene shows an electrical conductivity of $18.1 \mathrm{~S} \mathrm{~m}^{-1}$ and seebeck coefficient of $3228.8 \mu \mathrm{V} \mathrm{K}^{-1}$ as well as maintaining a thermal conductivity of $0.1 \mathrm{~W} \mathrm{~K}^{-1} \mathrm{~m}^{-1}$. This gives a ZT of 0.6 at $300 \mathrm{~K}$ which is significant for a bulk silicon based material and is similar to that of other thermoelectric materials such as $\mathrm{Mg}_{2} \mathrm{Si}, \mathrm{PbTe}$ and SiGe alloys.

\section{Introduction}

Over the past 20 years there has been increasing pressure for the development of technologies which take advantage of renewable energy sources. This is a result of the environmental impacts and future availability of fossil fuels. ${ }^{1}$ There has also been an emphasis on the recycling of waste energy (energy scavenging) to improve the efficiency of many industrial and commercial processes. $^{2}$

Thermoelectric materials are able to take advantage of wasted or unutilized heat sources, such as in furnaces, ${ }^{3}$ car exhausts, ${ }^{4}$ and solar cells. ${ }^{5}$ As a result thermoelectric materials have become an area of great interest. ${ }^{5 \mathrm{~d}, 6}$ These materials are able to convert a temperature gradient into electrical power, and vice versa without mechanical intervention. The power output from current commercial modules produced are however, modest, ${ }^{7}$ but the power generated from these devices can then be used elsewhere for low power applications, e.g. powering sensors or safety feedback loops.

The thermoelectric performance of a material can be expressed in terms of a figure of merit (ZT), given by the following expression: ${ }^{6 \mathrm{~b}}$

$Z T=\frac{\sigma S^{2}}{k} T$

where $\sigma$ is the electrical conductivity, $S$ is the Seebeck coefficient, $\kappa$ is thermal conductivity and $T$ is temperature. Commercial thermoelectric materials generally have a ZT value of around 1 .
The most common thermoelectric materials used today are based on bismuth telluride. ${ }^{6 \mathrm{a}} \mathrm{ZT}$ values of 2.4 at room temperature have been recorded for thin films, using bismuth telluride alloys, $\mathrm{Bi}_{2} \mathrm{Te}_{3} / \mathrm{Sb}_{2} \mathrm{Te}_{3} .{ }^{8}$ The major drawback with these materials is that the tellurium required to produce them is toxic and also expensive, due to its low abundance. As a consequence there has been a lot of focus on producing thermoelectric materials from alternative sources such as silicon, ${ }^{9}$ conductive polymers, ${ }^{10}$ and magnesium silicide. ${ }^{11}$

Silicon is a key material in the semiconductor electronics industry and as a result there is a well-established infrastructure based on these materials. ${ }^{12}$ Bulk silicon itself has a relatively high Seebeck coefficient $\left(-86 \mu \mathrm{V} \mathrm{K} \mathrm{K}^{-1}\right)$ and a high electrical conductivity when doping is optimized $\left(3030.3 \mathrm{~S} \mathrm{~cm}^{-1}\right)$, which results in a very high power factor. ${ }^{13}$ However, it also displays a very high thermal conductivity $87.3 \mathrm{~W} \mathrm{~m}^{-1} \mathrm{~K}^{-1}$ which results in a very low ZT of $0.008 .^{13}$

Silicon nanostructures show much lower thermal conductivities as a result of a more efficient boundary scattering of phonons, which can lead to a marked improvement to the figure of merit. ${ }^{14}$ For silicon nanowires, alteration of surface roughness, diameter and doping concentrations of nanowires has recently resulted in achieving a high $\mathrm{ZT}(\approx 1) .{ }^{9 \mathrm{~b}, \mathrm{c}, 15}$ Although it is worth noting, this was obtained from a single narrow nanowire, it is not yet demonstrated if the value can be translated into bulk materials.

Silicon nanowires show great potential as thermoelectric materials but preparation methods typically use harsh conditions or toxic and expensive reagents, such as trisilane, ${ }^{16}$ 
hydrogen fluoride and silver nitrate. ${ }^{17}$ It is possible to avoid such issues in the synthesis of silicon nanoparticles (SiNPs). ${ }^{18}$
Highly porous films or pellets of SiNPS Show exceptionally low thermal conductivity. ${ }^{10}$ These films also display low electrical

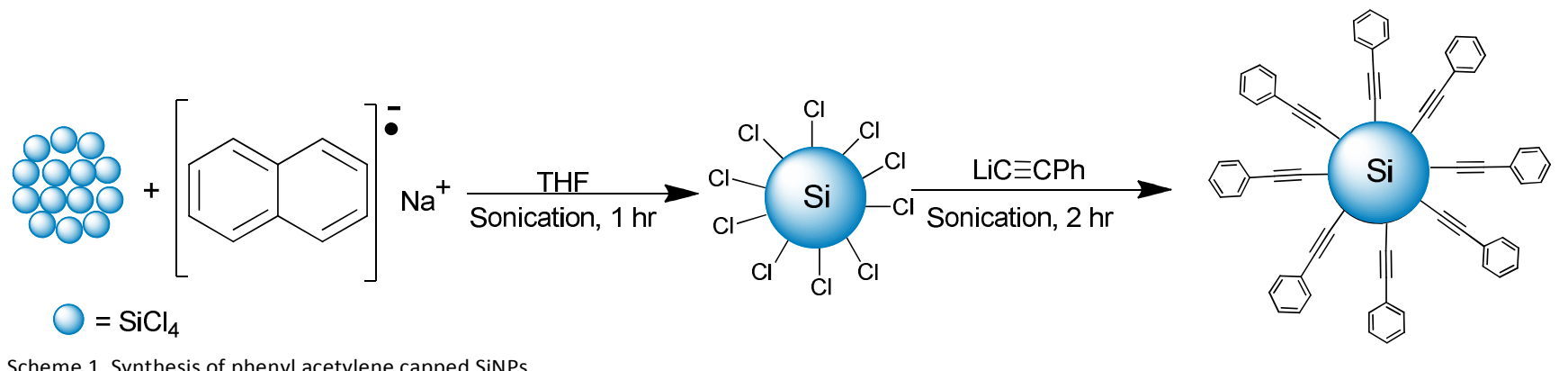

conductivity, due to the difficulty of transporting electrons across the junctions between SiNPs. ${ }^{19}$ Hydrogen terminated SiNPs require high temperature compaction or spark plasma sintering (SPS) to produce a conductive pellet. As a result crystal growth can be observed, significantly compromising the initial thermal conductivity advantages. ${ }^{13,20}$

Introducing suitable functionalization to the nanoscale silicon has been shown to be a method of maintaining the conductivity at the surface, ${ }^{21}$ and shows an improvement of the conductivity between two materials, acting as a conductive bridge. ${ }^{22}$ This study shows Si-Molecule-Metal junctions to be more conductive than a $\mathrm{Si}: \mathrm{H}-\mathrm{Hg}$ junction, which is attributed to the alignment of the ligands on the surface. More over the attachments of these molecules give an increased density of surface states of which have energy positions deep into the band gap, which act as slow traps that quench the surface photoconductivity. The use of a conjugated capping layer, such as phenylacetylene, could give an improved conductivity of a pellet presses at ambient temperature compared to that of a silicon based material of equivalent density and porosity.

There are issues with the attachment of such groups to a silicon surface, since the typical hydrosilylation method for SiNPs functionalization does not give desirable high surface coverage. ${ }^{23}$ This is due to the ability of such surface agents, once attached to quench the reactive species. ${ }^{24}$ However, an alternative method is the reaction of a suitable nucleophile with chloride terminated SiNPs. A well-documented synthesis for chloride terminated SiNPs is the reduction of $\mathrm{SiCl}_{4}$ using a suitable hydride free reducing agent such as sodium naphthalide or sodium silicide. ${ }^{25}$ Suitable nucleophiles which are subsequently introduced, include alcohols and organomatalic reagents. $^{26}$

In the following report phenylacetylene capped SiNPs are produced using a micelle reduction method. ${ }^{25,27}$ This entails the reduction of micelles of $\mathrm{SiCl}_{4}$ using sodium naphthalide to produce chlorine terminated SiNPs, followed by alkylation (functionalization) using lithium phenylacetylide as shown in Scheme 1. The resulting SiNPs were characterized using a combination of Fourier transform infrared spectroscopy (FTIR), ${ }^{1} \mathrm{H}-\mathrm{NMR},{ }^{13} \mathrm{C}-\mathrm{NMR}$, Transmission electron microscopy (TEM), $\mathrm{X}$-ray photoelectron spectroscopy (XPS), energy dispersive $\mathrm{x}$ - ray spectroscopy (EDX), thermo gravimetric analysis (TGA) and differential scanning calorimetry (DSC). The thermoelectric properties of a cold pressed pellet of phenylacetylene capped SiNPs were measured at room temperature. For this material a ZT of 0.6 was obtained, which is a high value for a bulk SiNPs pellet ${ }^{13,20}$ and is comparable to many thermoelectric materials. ${ }^{6 b, c, 28}$

\section{Experimental}

\section{Materials}

Silicon tetrachloride (Sigma, $99 \%$ ), lithium phenylacetylide (Sigma, $1 \mathrm{M}$ solution in tetrahydrofuran), sodium (Acros, 99.8 \%), naphthalene (Acros, $99 \%$ ), tetrahydrofuran (Fisher, 99.5 $\%$, dried over sodium/benzophenone and freshly distilled) and toluene (Fisher, $99 \%$ ).

\section{Synthesis of Phenylacetylene Capped SiNPs}

Silicon tetrachloride was dispersed in tetrahydrofuran by sonication for $15 \mathrm{mins}$ and a solution of freshly prepared sodium naphthalide $(70 \mathrm{~mL})$ was added. This was prepared by ultrasonication of sodium metal $(0.7 \mathrm{~g})$ and naphthalene $(2.9 \mathrm{~g})$ for $3 \mathrm{hr}$ in dry tetrahydrofuran $(70 \mathrm{~mL})$. After the addition of sodium naphthalide solution the mixture was placed in an ultrasonic bath for $1 \mathrm{hr}$ to give a dark brown suspension. To this a solution of lithium phenylacetylide $(5 \mathrm{~mL}, 1 \mathrm{M}$ solution in tetrahydrofuran) was added followed by further ultrasonication for $2 \mathrm{hr}$. All precipitate was allowed to settle, the clear liquid was decanted off and all solvent was removed in vacou. The resulting solid was then heated to $373 \mathrm{~K}$ under reduced pressure to remove the residual naphthalene. The resulting solid was redissolved in toluene, washed with water to remove any remaining lithium chloride; the organic layer was dried to give phenylacetylene capped SiNPs, as an orange crystalline solid.

\section{FTIR}

The FTIR spectrum was collected using a Perkin-Elmer Spectrum 100 ATR FTIR spectrometer. The background was corrected by subtracting a spectrum of the clean crystal.

\section{H-NMR and 13C-NMR}


Measurements of sample dissolved in $\mathrm{CDCl} 3$ were taken using a Bruker $500 \mathrm{MHz}$ NMR spectrometer. These samples were measured relative to chloroform from the lock solvent $\left(\mathrm{CDCl}_{3}\right)$.

\section{XPS}

XPS analysis was carried out on a K-Alpha XPS instrument (Thermo Scientific, East Grinstead, UK). A monochromatic Al Ka X-ray source $(1486.6 \mathrm{eV}$ ) was used with a spot size of 400 $\mathrm{mm}$ diameter. A pass energy of $200 \mathrm{eV}$ and step size of $0.4 \mathrm{eV}$ was used for survey spectra. A pass energy of $40 \mathrm{eV}$ and step size of $0.1 \mathrm{eV}$ was used for high resolution spectra.

\section{TEM}

TEM micrographs were taken using a JEOL JEM2000 transmission electron microscope. TEM samples were prepared by drop casting a solution of phenylacetylene capped SiNPs in chloroform onto a 200 mesh copper grid with a holey carbon film. The solvent was evaporated and TEM micrographs were taken at different spots of the grid. The mean diameter and standard deviation were acquired by the measurement of 100 nanoparticles manually from images of different spots on the grid.

\section{TGA and DSC}

TGA and DSC analysis was performed using a METTLERTOLEDO TGA-1. The solid sample (approx. $5 \mathrm{mg}$ ) was placed in a $40 \mu \mathrm{L}$ aluminum pan and measurements were taken during the heating of the sample from 300 to $773 \mathrm{~K}$. The background measurements were taken while heating the empty pan over the same temperature range.

\section{Preparation of pellet and measurement of thermoelectric properties}

A dry powder of the sample was pressed uniaxially using a hydraulic press and die into a $13 \mathrm{~mm}$ diameter pellet of thickness $1-2 \mathrm{~mm}$ by applying the maximum pressure of 800 $\mathrm{MPa}$ for $3 \mathrm{~min}$. The pressing was carried out at room temperature.

The Seebeck coefficient was measured at room temperature using a hot probe apparatus. ${ }^{29}$ A four probe DC current equipment was used to measure the room temperature electrical resistivity. A current $\mathrm{I}=10 \mathrm{~mA}$ was applied between two probes and the voltage difference $\mathrm{V}$ generated at the other two probes was measured. The resistivity $\mathrm{R}$ was calculated using

$R=\frac{V}{I} \times F \times C \times d$

where $\mathrm{F}$ and $\mathrm{C}$ are geometrical correction factors and $\mathrm{d}$ the pellet thickness. In both measurements the data was taken at different regions of the pellet. The resistivity reported here is the average of two measurements with applied electrical current in two opposite directions. The contacts are spring loaded pressure contact. The influence of the parasitic capacitance is ignored here because the measurement was carried out under DC condition as thermoelectric devices operate in DC mode.
Thermal diffusivity measurements were $10.1039 / \mathrm{C} 4 \mathrm{FD} 00109 \mathrm{~F}$ and $423 \mathrm{~K}$ using an Anter Flashline 3000 instrument. Measurements were made on pellets of phenylacetylene capped silicon nanoparticles. This instrument determines both the thermal diffusivity $(\alpha)$ and the heat capacity $\left(C_{p}\right)$ of the sample. The thermal conductivity $(\kappa)$ is calculated from the relationship:

$\kappa=\alpha C_{p} \rho$

where $\rho$ is the sample density. For the determination of the heat capacity, side-by-side testing of a reference material, PyroceramTM 9606, of known heat capacity, was carried out. The procedure used for the determination of the heat capacity has been described in detail. ${ }^{30}$ Efforts to extend the measurements to higher temperatures were unsuccessful as the sample pellet deformed.

\section{Results and Discussion}

The FTIR spectrum (Figure 1) shows the peaks expected for the phenylacetylene functionalization of SiNPs. The spectrum shows peaks at $3051 \mathrm{~cm}^{-1}$, representative of aromatic $\mathrm{C}-\mathrm{H}$, and at both 1596 and $1487 \mathrm{~cm}^{-1}$ which is representative of aromatic C-C bonds. Also clearly visible is the sharp peak at $2161 \mathrm{~cm}^{-1}$, which is characteristic of the $\mathrm{C} \equiv \mathrm{C}$, which, when paired with the absence of a sharp peak at $3300 \mathrm{~cm}^{-1}$, suggests that the terminal side of the alkyne is attached to silicon. This is also supported by the $\mathrm{Si}-\mathrm{C} \equiv \mathrm{C}$ peaks at 1441 and $1221 \mathrm{~cm}^{-1}$. The Si-O peak between 1100 and $1000 \mathrm{~cm}^{-1}$ is observable but is broad and weak when compared with the sharp peaks at 1068 and 1026 $\mathrm{cm}^{-1}$ which are also characteristic of phenylacetylene.

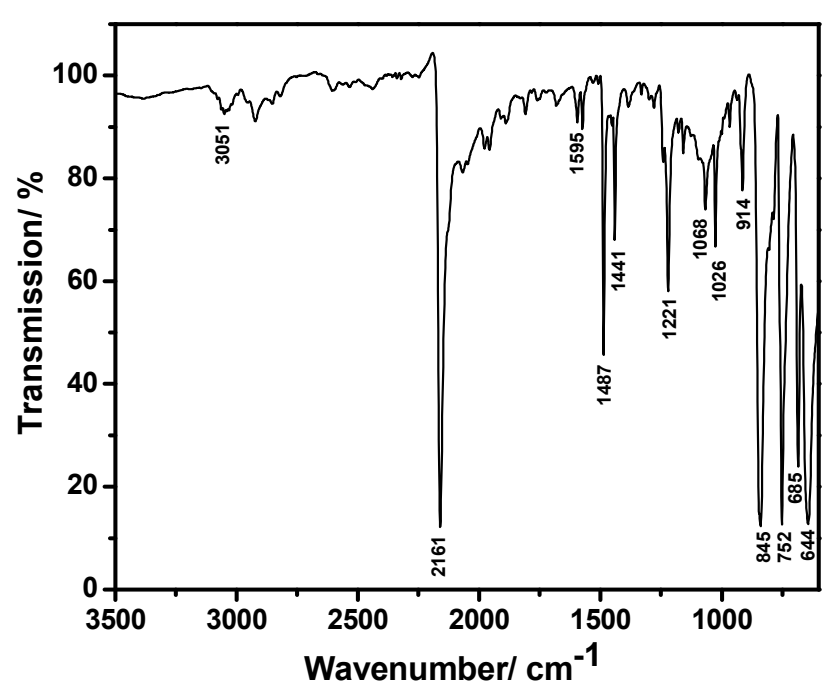

Figure 1. FTIR spectrum of phenylacetylene capped SiNPs.

Further evidence of phenylacetylene capping can also be observed in the ${ }^{1} \mathrm{H}-\mathrm{NMR}$ (Figure 2a) and ${ }^{13} \mathrm{C}-\mathrm{NMR}$ (Figure 2b) spectra. ${ }^{1} \mathrm{H}-\mathrm{NMR}$ shows two multiplet peaks at 7.53 and 7.30 ppm with an integration of 2:3 respectively, characteristic of the phenyl protons of phenylacetylene. Also, there was no presence of the peak for a proton on the alkyne at $3 \mathrm{ppm}$. These features 
are evidence of the molecule being bound to the SiNPs. The ${ }^{13} \mathrm{C}-\mathrm{NMR}$ shows the four peaks for the phenyl carbons at 132 , 129,128 and $121 \mathrm{ppm}$ and 2 peaks for the alkyne at 106 and 86 ppm, characteristic of the carbon closest to the phenyl and the silicon respectively.
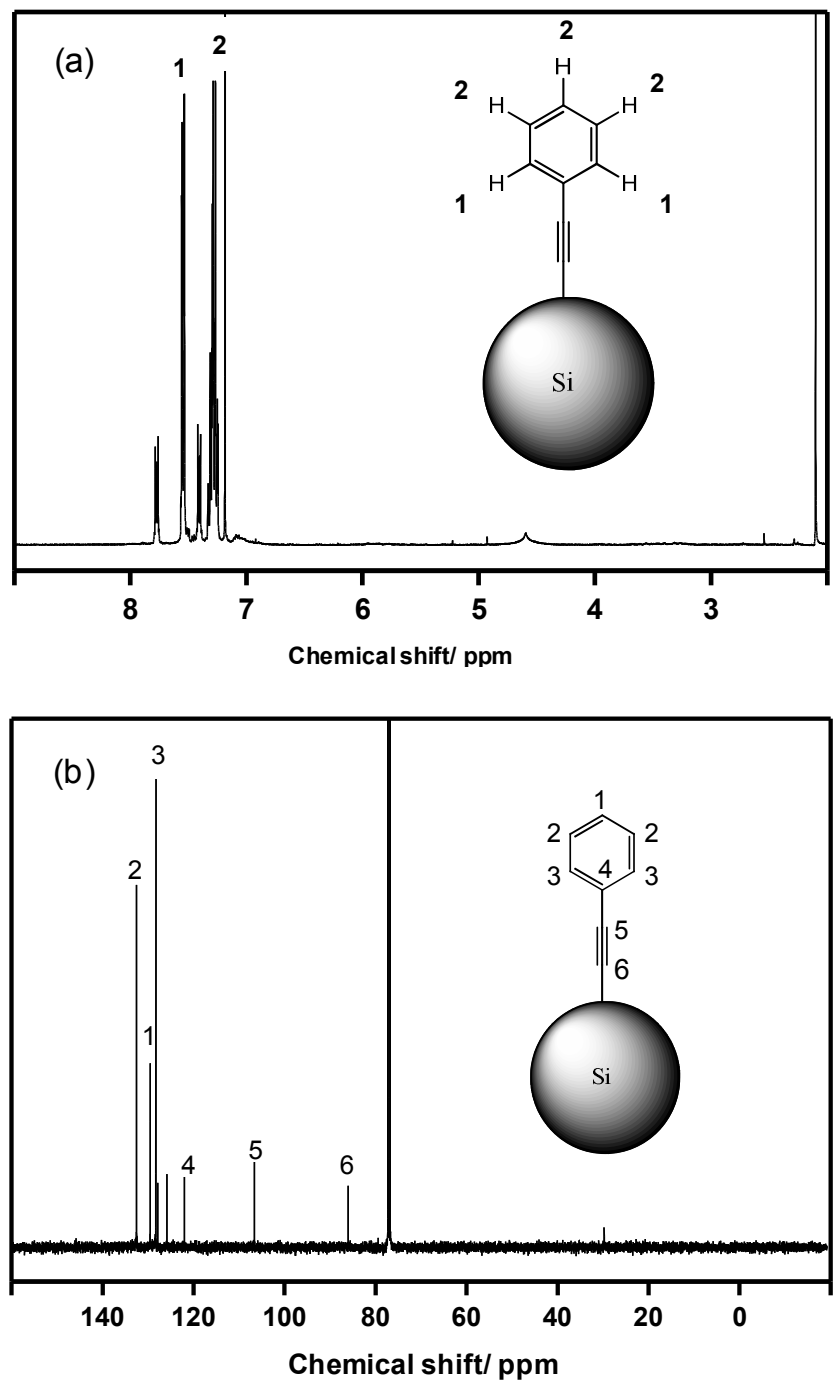

Figure 2. NMR spectrum of phenylacetylene capped SiNPs: (a) 1H-NMR; (b) 13CNMR.

The TEM image shows that spherical SiNPs have been synthesized (Figure 3). The SiNPs show a wide distribution of particle diameters (inset Figure 3). The mean diameter of the nanoparticles produced is $6 \mathrm{~nm}$ with a standard deviation of 1 $\mathrm{nm}$, obtained by analyzing 100 particles from different regions of the grid. The high standard deviation and wide size distribution can be explained by the lack of controlling influence on the micelle size, i.e. lack of use of surfactants. ${ }^{18 a}$

The SiNPs are rich in both silicon (inset Figure 4) and carbon. This can be observed from the XPS survey spectrum of the SiNPs (Figure 4). Moderate levels of oxygen can also been seen in Figure 4. However, lower levels of oxygen were observed on EDX (Supporting Information). This increased oxygen level observed in XPS is typical of X-ray induced OFDo1dation of SiNPs. ${ }^{31}$ Additionally XPS does not show any signals from elemental impurities of potential byproducts: sodium, lithium and chlorine.

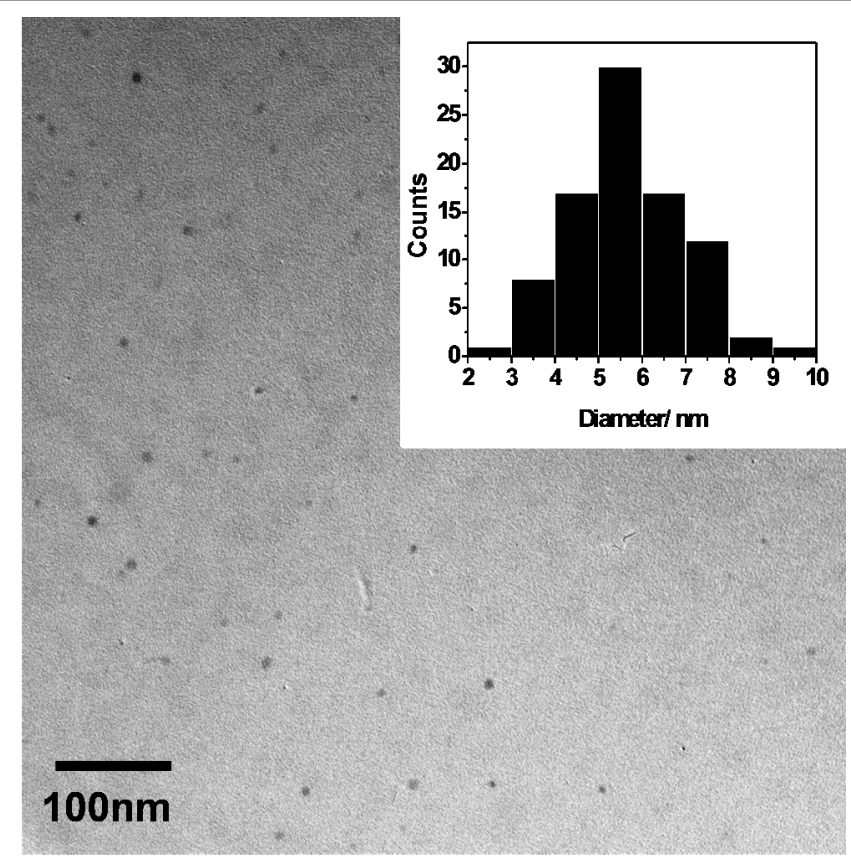

Figure 3. TEM image and size distribution of phenylacetylene capped silicon nanoparticles. Inset, size distribution of silicon nanoparticles.

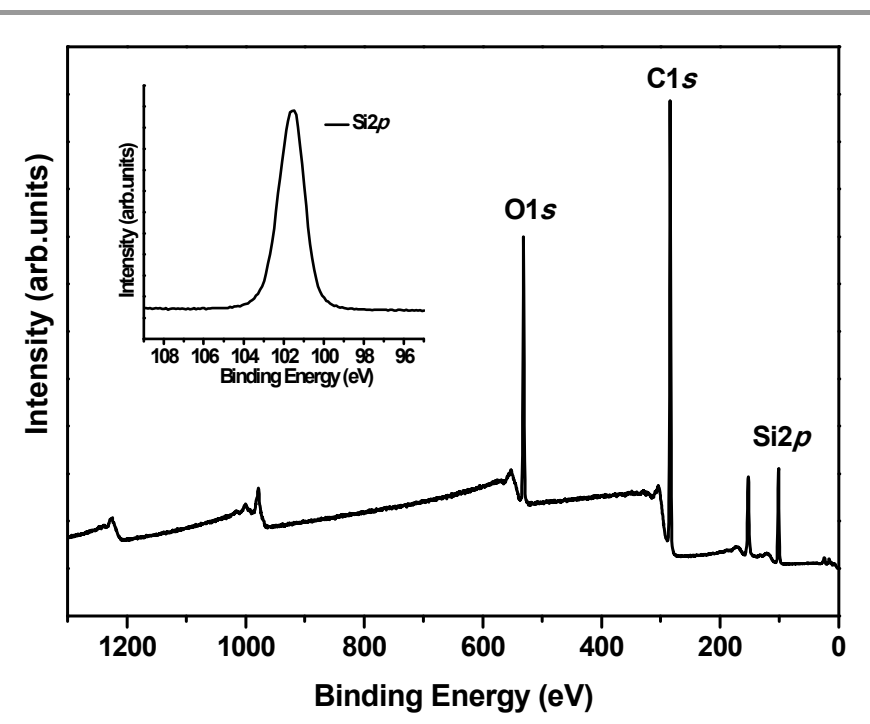

Figure 4. XPS survey spectrum for phenylacetylene capped silicon nanoparticles. Inset, high resolution XPS spectrum of silicon signal.

The DSC trace (Figure 5) begins at a flat level of heat flow before a negative peak can be observed at $445 \mathrm{~K}$ representing a solid-liquid phase transition. Meanwhile the weight on the TGA trace (Figure 5) remains close to $100 \%$. After this feature the heat flow into the sample begins to decrease relatively rapidly, which suggests that the sample is beginning to undergo degradation. The DSC trace reaches a maximum at $593 \mathrm{~K}$, where there is no net flow of heat into or out of the sample 
showing completion of the initial degradation. After $593 \mathrm{~K}$ the heat flow into the sample increases again, the TGA trace shows that the weight continues to decrease; becoming more rapid above $700 \mathrm{~K}$. This is likely to be due to continued degradation. The DSC and TGA traces are significant in that they show to what temperature a material produced from phenylacetylene capped SiNPs can be used or tested. The observation of a solid to liquid phase transition at $448 \mathrm{~K}$ suggests that the material could only be used up to approx $420 \mathrm{~K}$, before the melting process begins warping the material. Also it should be noted that this limits the temperatures at which material can be processed as significant softening occurs a moderately low temperatures. Thus, the material would be suitable for low temperature thermoelectric applications only. Examples include: energy scavenging from hot water/cooling water outflow from a plant or thermal spring, or the conversion of heat from light harvesting.

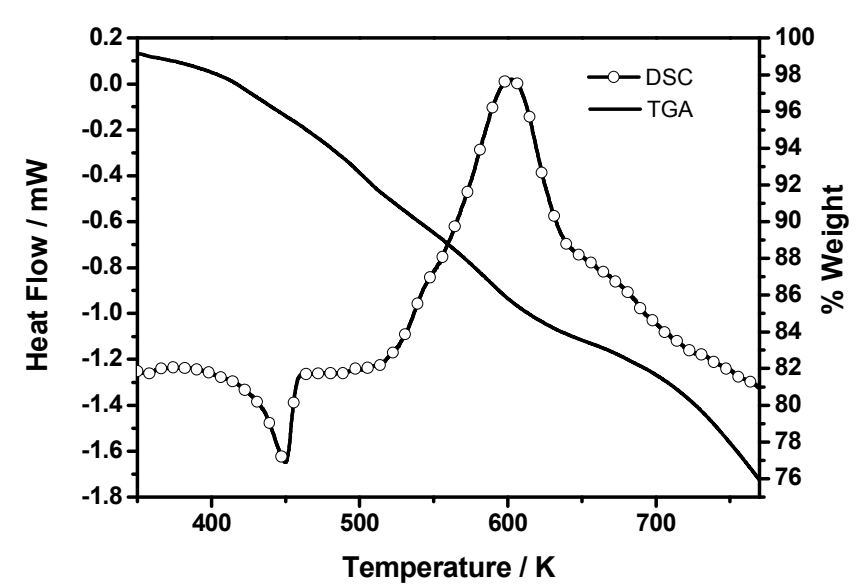

Figure 5. TGA and DSC analysis of phenylacetylene functionalised silicon nanoparticles.

The thermoelectric measurements are given in Table 1. The measurements obtained at ambient temperature show that the material produced has an exceptionally high Seebeck coefficient and low thermal conductivity. These two values offset the low electrical conductivity in the equation for the figure of merit $(\mathrm{ZT})$. The thermal conductivity was measured at $373 \mathrm{~K}$ and $423 \mathrm{~K}$, and the values were 0.15 and $0.19 \mathrm{~W} \mathrm{~m}^{-1} \mathrm{~K}^{-1}$ respectively. The listed value in Table 1 is an extrapolated value expressed to one decimal place. This gives an ambient temperature $\mathrm{ZT}$ of 0.6 . This value is comparative to other materials under investigation in thermoelectrics. ${ }^{9 b, c}$

Table 1. Thermoelectric measurements of a pellet of phenylacetylene capped silicon nanoparticles at room temperature.

\begin{tabular}{|c|c|c|c|c|}
\hline$\rho\left[\mathrm{kg} \mathrm{m}^{-3}\right]$ & $\sigma\left[\mathrm{S} \mathrm{m}^{-1}\right]$ & $\mathrm{S}\left[\mu \mathrm{V} \mathrm{K}^{-1}\right]$ & $\kappa\left[\mathrm{W} \mathrm{m}^{-1} \mathrm{~K}^{-1}\right]$ & $\mathrm{ZT}$ \\
\hline 1022.0 & 18.1 & 3228.8 & 0.1 & 0.6 \\
\hline
\end{tabular}

The low thermal conductivity values may be in part due to the low density. Because the samples were simply cold pressed, the density might be well below the theoretical (crystallographic) value. The effect of densification on $\kappa$ is of the form below: ${ }^{32}$

$\frac{k_{\text {porous }}}{k_{\text {dense }}}=1-\frac{4}{3} \varphi$

where $\varphi$ is the porosity defined as

$\varphi=1-\frac{\rho_{\text {porous }}}{\rho_{\text {dense }}}$

Unfortunately, given the presence of the organic capping agents, the calculation of the theoretical density is less/not obvious. If the organic component is neglected, then the density is expected to be that of silicon, which is $2329 \mathrm{~kg} \mathrm{~m}^{-3}$. The measured density is approximately $1022 \mathrm{~kg} \mathrm{~m}^{-3}$. This suggests, compared to pure silicon, the sample is approximately $44 \%$ dense. Of course this is a gross approximation as it ignores the organic component completely, but it does suggest that the sample is quite porous and that $\kappa$ is well below that of the dense material.

Relative to other silicon materials of similar porosity, the electrical conductivity of a pellet of phenylacetylene SiNPs is significantly large. ${ }^{19 b}$, c, 33 However, when compared with materials typically involved in thermoelectric applications the electrical conductivity is low. ${ }^{5,}$, 6 This conductivity could however be improved and optimized through the introduction of charge carriers, which can be achieved by doping the SiNPs and/or doping the ligands. Both of these should be understood in order to optimize this material.

The magnitude of the Seebeck coefficient is much higher than that which is documented for other silicon based materials. ${ }^{13,} 34$ This can be attributed to the low carrier concentration discussed above. If the charge carrier concentration is optimized the Seebeck coefficient would be expected to be reduced by a factor of 10 to 100 , based on previous work regarding silicon based materials. ${ }^{13,34}$ Optimization of doping would mean that a compromise between the magnitude of the electrical conductivity and Seebeck coefficient will need to be made, which in practical terms would make for a more useful material but may alter the ZT positively or negatively.

\section{CONCLUSION}

Surface analysis of phenylacetylene functionalized SiNPs shows a surface coverage considerably higher than that previously documented for capping with similar molecules using hydrosilylation based methods. The thermal stability shows that the material produced, when cold pressed undergoes a phase transition at $448 \mathrm{~K}$, so would only be suitable to be used as a thermoelectric material up to $420 \mathrm{~K}$. The thermoelectric performance at ambient temperature is comparable to other silicon based thermoelectric materials, such as silicon nanowires, where a $\mathrm{ZT} \approx 1$ was obtained from a single narrow nanowire. Although it is not yet demonstrated if the ZT value can be translated into bulk materials. On the other 
hand, phenylacetylene capped silicon nanoparticles have been readily compacted into bulk materials with a respectable ZT of 0.6 prior to optimization of the electrical conductivity by doping. This is a high $\mathrm{ZT}$ value compared to other silicon based bulk materials and is comparable to other better established thermoelectric materials such as $\mathrm{Mg}_{2} \mathrm{Si}$ and $\mathrm{SiGe}$ Alloys. This result shows a significant step toward silicon based thermoelectrics.

\section{Acknowledgements}

SPA is grateful to an Industry CASE studentship sponsored by the UK Engineering and Physical Science Research Council (ESPRC), and European Thermodynamics Ltd. X-ray photoelectron spectra were obtained at the National EPSRC XPS User's Service (NEXUS) at Newcastle University, an EPSRC Mid-Range Facility.

\section{Notes and references}

${ }^{a}$ School of Chemistry, University of East Anglia, Norwich NR4 7TJ, UK. Fax: +441603592003; Tel: +441603593146

${ }^{b}$ School of Engineering, Cardiff University, Cardiff CF24 3AA, UK.

${ }^{c}$ Institute of Chemical Sciences, Heriot Watt University, Edinburgh EH14 4AS, UK.

$d$ Advance Materials Laboratory, Department of Chemistry, Fudan University, Shanghai 200433, P. R. China.

Electronic Supplementary Information (ESI) available: EDX Spectrum. See DOI: $10.1039 / \mathrm{b} 000000 \mathrm{x} /$

1. (a) Schneider, S. H., The Greenhouse Effect: Science and Policy. Science 1989, 243 (4892), 771-781; (b) Solomon, S.; Plattner, G.-K.; Knutti, R.; Friedlingstein, P., Irreversible climate change due to carbon dioxide emissions. Proceedings of the National Academy of Sciences 2009, 106 (6), 1704-1709.

2. Qin, Y.; Wang, X.; Wang, Z. L., Microfibre-nanowire hybrid structure for energy scavenging. Nature 2008, 451 (7180), 809-U5.

3. Kajikawa, T., Approach to the Practical Use of Thermoelectric Power Generation. J. Electron. Mater. 2009, 38 (7), 1083-1088.

4. Yang, J.; Stabler, F., Automotive Applications of Thermoelectric Materials. J. Electron. Mater. 2009, 38 (7), 1245-1251.

5. (a) Kraemer, D.; Poudel, B.; Feng, H.-P.; Caylor, J. C.; Yu, B.; Yan, X.; Ma, Y.; Wang, X.; Wang, D.; Muto, A.; McEnaney, K.; Chiesa, M.; Ren, Z.; Chen, G., High-performance flat-panel solar thermoelectric generators with high thermal concentration. Nat Mater 2011, 10 (7), $532-$ 538; (b) Lertsatitthanakorn, C.; Rungsiyopas, M.; Therdyothin, A.; Soponronnarit, S., Performance Study of a Double-Pass Thermoelectric Solar Air Collector with Flat-Plate Reflectors. J. Electron. Mater. 2012, 41 (6), 999-1003; (c) Minnich, A. J.; Dresselhaus, M. S.; Ren, Z. F.; Chen, G., Bulk nanostructured thermoelectric materials: current research and future prospects. Energy \& Environmental Science 2009, 2 (5), 466479; (d) Tritt, T. M.; Subramanian, M. A., Thermoelectric materials, phenomena, and applications: A bird's eye view. MRS Bull. 2006, 31 (3), 188-194.

6. (a) Poudel, B.; Hao, Q.; Ma, Y.; Lan, Y.; Minnich, A.; Yu, B.; Yan, X.; Wang, D.; Muto, A.; Vashaee, D.; Chen, X.; Liu, J.; Dresselhaus, M.
Journal Name

View Article Online

S.; Chen, G.; Ren, Z., High-Thermoelectric Performance of Nanostructured Bismuth Antimony Telluride Bulk Alloys. Science 2008, 320 (5876), 634-638; (b) Kanatzidis, M. G., Nanostructured Thermoelectrics: The New Paradigm?†. Chemistry of Materials 2009, 22 (3), 648-659; (c) Dresselhaus, M. S.; Chen, G.; Tang, M. Y.; Yang, R. G.; Lee, H.; Wang, D. Z.; Ren, Z. F.; Fleurial, J. P.; Gogna, P., New directions for low-dimensional thermoelectric materials. Adv. Mater. 2007, 19 (8), 1043-1053.

7. Funahashi, R.; Urata, S.; Mizuno, K.; Kouuchi, T.; Mikami, M., $\mathrm{Ca} 2.7 \mathrm{Bi} 0.3 \mathrm{Co} 4 \mathrm{O} 9 / \mathrm{La} 0.9 \mathrm{Bi0} .1 \mathrm{NiO} 3$ thermoelectric devices with high output power density. Appl. Phys. Lett. 2004, 85 (6), 1036-1038.

8. Venkatasubramanian, R.; Siivola, E.; Colpitts, T.; O'Quinn, B., Thinfilm thermoelectric devices with high room-temperature figures of merit. Nature 2001, 413 (6856), 597-602.

9. (a) Tang, J.; Wang, H.-T.; Lee, D. H.; Fardy, M.; Huo, Z.; Russell, T. P.; Yang, P., Holey Silicon as an Efficient Thermoelectric Material. Nano Lett. 2010, 10 (10), 4279-4283; (b) Boukai, A. I.; Bunimovich, Y.; TahirKheli, J.; Yu, J. K.; Goddard, W. A.; Heath, J. R., Silicon nanowires as efficient thermoelectric materials. Nature 2008, 451, 168-171; (c) Hochbaum, A. I.; Chen, R. K.; Delgado, R. D.; Liang, W. J.; Garnett, E. C.; Najarian, M.; Majumdar, A.; Yang, P. D., Enhanced thermoelectric performance of rough silicon nanowires. Nature 2008, 451, 163-167.

10. Bubnova, O.; Crispin, X., Towards polymer-based organic thermoelectric generators. Energy Environ. Sci. 2012, 5 (11), 9345-9362.

11. Zaitsev, V. K.; Fedorov, M. I.; Gurieva, E. A.; Eremin, I. S.; Konstantinov, P. P.; Samunin, A. Y.; Vedernikov, M. V., Highly effective Mg2Si1-xSnx thermoelectrics. Phys. Rev. B 2006, 74 (4), 045207.

12. Kang, Z.; Liu, Y.; Lee, S.-T., Small-sized silicon nanoparticles: new nanolights and nanocatalysts. Nanoscale 2011, 3 (3), 777-791.

13. Bux, S. K.; Blair, R. G.; Gogna, P. K.; Lee, H.; Chen, G.; Dresselhaus, M. S.; Kaner, R. B.; Fleurial, J.-P., Nanostructured Bulk Silicon as an Effective Thermoelectric Material. Advanced Functional Materials 2009, 19 (15), 2445-2452.

14. Sharp, J. W.; Poon, S. J.; Goldsmid, H. J., Boundary Scattering and the Thermoelectric Figure of Merit. physica status solidi (a) 2001, 187 (2), 507-516.

15. Dasgupta, N. P.; Sun, J.; Liu, C.; Brittman, S.; Andrews, S. C.; Lim, J.; Gao, H.; Yan, R.; Yang, P., 25th Anniversary Article: Semiconductor Nanowires - Synthesis, Characterization, and Applications. Advanced Materials 2014, 26 (14), 2137-2184.

16. (a) Heitsch, A. T.; Akhavan, V. A.; Korgel, B. A., Rapid SFLS Synthesis of Si Nanowires Using Trisilane with In situ Alkyl-Amine Passivation. Chemistry of Materials 2011, 23 (11), 2697-2699; (b) Chen, H.; Xu, J.; Chen, P.-c.; Fang, X.; Qiu, J.; Fu, Y.; Zhou, C., Bulk Synthesis of Crystalline and Crystalline Core/Amorphous Shell Silicon Nanowires and Their Application for Energy Storage. ACS Nano 2011, 5 (10), 83838390.

17. Peng, K.; Xu, Y.; Wu, Y.; Yan, Y.; Lee, S.-T.; Zhu, J., Aligned Single-Crystalline Si Nanowire Arrays for Photovoltaic Applications. Small 2005, 1 (11), 1062-1067.

18. (a) Wang, J.; Sun, S.; Peng, F.; Cao, L.; Sun, L., Efficient one-pot synthesis of highly photoluminescent alkyl-functionalised silicon nanocrystals. Chemical Communications 2011, 47 (17), 4941; (b) Wilcoxon, J. P.; Samara, G. A., Tailorable, visible light emission from silicon nanocrystals. Appl. Phys. Lett. 1999, 74 (21), 3164-3166. 
19. (a) Khan, Z. H., Electrical and optical properties of thin film of amorphous silicon nanoparticles. Applied Surface Science 2009, 255 (21), 8874-8878; (b) Aroutiounian, V. M.; Ghulinyan, M. Z., Electrical conductivity mechanisms in porous silicon. physica status solidi (a) 2003, 197 (2), 462-466; (c) Bouaïcha, M.; Khardani, M.; Bessaïs, B., Evaluation of the electrical conductivity of nano-porous silicon from photoluminescence and particle size distribution. Materials Science and Engineering: B 2008, 147 (2-3), 235-238; (d) More, P. V.; Jeong, S.; Lee, J.-Y.; Seo, Y.-H.; Ryu, B.-H.; Choi, Y., Printable electronicscompatible silicon nanoparticles prepared by the facile decomposition of SiS2 and their application in a back-to-back Schottky diode. Journal of Materials Chemistry 2012, 22 (44), 23553-23560.

20. Zhu, G. H.; Lee, H.; Lan, Y. C.; Wang, X. W.; Joshi, G.; Wang, D. Z.; Yang, J.; Vashaee, D.; Guilbert, H.; Pillitteri, A.; Dresselhaus, M. S.; Chen, G.; Ren, Z. F., Increased Phonon Scattering by Nanograins and Point Defects in Nanostructured Silicon with a Low Concentration of Germanium. Physical Review Letters 2009, 102 (19), 196803.

21. Saito, N.; Hayashi, K.; Sugimura, H.; Takai, O., Microstructured $\pi$ Conjugated Organic Monolayer Covalently Attached to Silicon. Langmuir 2003, 19 (26), 10632-10634.

22. Hiremath, R. K.; Mulimani, B. G.; Rabinal, M. K.; Khazi, I. M., Electrical characterization of a phenylacetylene-modified silicon surface via mercury probe. Journal of Physics: Condensed Matter 2007, 19 (44), 446003.

23. Kelly, J. A.; Shukaliak, A. M.; Fleischauer, M. D.; Veinot, J. G. C., Size-dependent reactivity in hydrosilylation of silicon nanocrystals. $J$. Am. Chem. Soc. 2011, 133 (46), 19015-6.

24. (a) Stewart, M. P.; Buriak, J. M., Exciton-Mediated Hydrosilylation on Photoluminescent Nanocrystalline Silicon. J. Am. Chem. Soc. 2001, 123 (32), 7821-7830; (b) Kelly, J. A.; Veinot, J. G. C., An Investigation into Near-UV Hydrosilylation of Freestanding Silicon Nanocrystals. ACS Nano 2010, 4 (8), 4645-4656.

25. (a) Baldwin, R. K.; Pettigrew, K. A.; Garno, J. C.; Power, P. P.; Liu, G.-y.; Kauzlarich, S. M., Room Temperature Solution Synthesis of AlkylCapped Tetrahedral Shaped Silicon Nanocrystals. J. Am. Chem. Soc. 2002, 124 (7), 1150-1151; (b) Pettigrew, K. A.; Liu, Q.; Power, P. P.; Kauzlarich, S. M., Solution synthesis of alkyl- and alkyl/alkoxy-capped silicon nanoparticles via oxidation of $\mathrm{Mg} 2 \mathrm{Si}$. Chem. Mater. 2003, 15 (21), 4005-4011.

26. (a) Veinot, J. G. C., Synthesis, surface functionalization, and properties of freestanding silicon nanocrystals. Chemical Communications 2006, (40), 4160-4168; (b) Buriak, J. M., Organometallic chemistry on silicon and germanium surfaces. Chem. Rev. 2002, 102 (5), 1271-1308.

27. Ashby, S.; Thomas, J.; Coxon, P.; Bilton, M.; Brydson, R.; Pennycook, T.; Chao, Y., The effect of alkyl chain length on the level of capping of silicon nanoparticles produced by a one-pot synthesis route based on the chemical reduction of micelle. J. Nanopart. Res. 2013, 15 (2), 1425 .

28. Zaitsev, V. K.; Fedorov, M. I.; Gurieva, E. A.; Eremin, I. S.; Konstantinov, P. P.; Samunin, A. Y.; Vedernikov, M. V., Highly effective Mg2Si1-xSnx thermoelectrics. Phys. Rev. B 2006, 74 (4).

29. Golan, G.; Axelevitch, A.; Gorenstein, B.; Manevych, V., Hot-Probe method for evaluation of impurities concentration in semiconductors. Microelectronics Journal 2006, 37 (9), 910-915.
30. (a) Gaal, P. S.; Apostolescu, S. P. 2002; (b) Thermitus, M.-A.; Gaal, P. S. In Proceedings of the 24th International Thermal Conductivity Conference, 24th International Thermal Conductivity Conference, Lancaster, Uher, C.; Morelli, D., Eds. Technomic Publishing Co.: Lancaster, 1997; p 340.

31. (a) Chao, Y.; Wang, Q.; Pietzsch, A.; Hennies, F.; Ni, H., Soft X-ray induced oxidation on acrylic acid grafted luminescent silicon quantum dots in ultrahigh vacuum. Phys. Status Solidi A-Appl. Mat. 2011, 208 (10), 2424-2429; (b) Coxon, P. R.; Ashby, S. P.; Frogley, M. D.; Chao, Y., Thermal evaporation and x-ray photostability of dodecyl-passivated silicon nanoparticles. J. Phys. D: Appl. Phys. 2012, 45 (35), 355303.

32. (a) Klemens, P. G., Thermal conductivity of inhomogeneous media. High Temperatures - High Pressures 1991, 23, 241-248; (b) Schlichting, K. W.; Padture, N. P.; Klemens, P. G., Thermal conductivity of dense and porous yttria-stabilized zirconia. J. Mater. Sci. 2001, 36 (12), 3003-3010. 33. Hochbaum, A. I.; Chen, R. K.; Delgado, R. D.; Liang, W. J.; Garnett, E. C.; Najarian, M.; Majumdar, A.; Yang, P. D., Enhanced thermoelectric performance of rough silicon nanowires. Nature 2008, 451 (7175), 163U5.

34. Boukai, A. I.; Bunimovich, Y.; Tahir-Kheli, J.; Yu, J. K.; Goddard, W. A.; Heath, J. R., Silicon nanowires as efficient thermoelectric materials. Nature 2008, 451 (7175), 168-171. 